\title{
Accurate State Estimation and Tracking of a Non-Cooperative Target Vehicle
}

\author{
Julie K. Thienel ${ }^{*}$ \\ U.S. Naval Academy, Department of Aerospace Engineering \\ Annapolis, Maryland, 21402, USA \\ John M. VanEepoel ${ }^{\dagger}$ \\ NASA Goddard Space Flight Center, Flight Dynamics Analysis Branch \\ Greenbelt, Maryland, 20771, USA \\ Robert M. Sanner \\ University of Maryland, Aerospace Engineering Department, College Park, Maryland, 20742, USA
}

\begin{abstract}
Autonomous space rendezvous scenarios require knowledge of the target vehicle state in order to safely dock with the chaser vehicle. Ideally, the target vehicle state information is derived from telemetered data, or with the use of known tracking points on the target vehicle. However, if the target vehicle is non-cooperative and does not have the ability to maintain attitude control, or transmit attitude knowledge, the docking becomes more challenging. This work presents a nonlinear approach for estimating the body rates of a non-cooperative target vehicle, and coupling this estimation to a tracking control scheme. The approach is tested with the robotic servicing mission concept for the Hubble Space Telescope (HST). Such a mission would not only require estimates of the HST attitude and rates, but also precision control to achieve the desired rate and maintain the orientation to successfully dock with HST.
\end{abstract}

\section{INTRODUCTION}

Vehicles designed to rendezvous and dock with existing space missions are being considered for a number of scenarios. In-space assembly is considered a fundamental requirement to accomplish space exploration goals. ${ }^{1,2}$ 'Space Tug' concepts are being designed to provide servicing and refuelling for in-flight missions. ${ }^{3,4}$ Kimura, et al present a demonstration mission for on-orbit maintenance of satellites. ${ }^{5}$ In 2004 former NASA Administrator Sean O'Keefe asked the Hubble Space Telescope (HST) program to investigate robotic servicing of the HST to extend the science life. ${ }^{6}$ In all cases, the chase vehicle must have knowledge of the target vehicle state and must perform precise control in order to safely dock with the target vehicle. When the target vehicle is non-cooperative, meeting the knowledge and control requirements becomes much more challenging.

This work applies an approach to estimate the attitude and rates of a non-cooperative target vehicle, and then uses the attitude and rate estimates as the desired state of the chase vehicle tracking control algorithm. The target vehicle attitude estimate is assumed to be provided by a vision or feature-based sensor. The target rate is determined with the nonlinear estimation approach presented in reference 7 . The estimator is exponentially stable in the absence of any measurement errors, and remains robust to bounded perturbations resulting from uncertainties in the measured target attitude. The estimator provides the desired rate for the nonlinear passivity-based control scheme presented in reference 8 . The nonlinear controller is asymptotically stable in the absence of any disturbances. The actual attitude of the chase vehicle is assumed to be available from an accurate sensor such as a star tracker. The chase vehicle rates are provided by calibrated gyros.

The stability of the nonlinear control algorithm, given bounded estimates of the desired state of the chase vehicle, is explored through simulations. We consider the effects of additional error sources, such as second

\footnotetext{
*Aerospace Engineer, thienel@usna.edu, phone:410-293-6400, and AIAA Member.

$\dagger$ Aerospace Engineer, john.m.vaneepoel@nasa.gov, phone:301-286-9216, and AIAA member.

¥Professor of Aerospace Engineering, rmsanner@eng.umd.edu, phone:301-405-1928, and AIAA member.
} 
to here as the Hubble Robotic Vehicle (HRV), is used as the test scenario. The HRV must be capable of docking with HST, regardless of the orientation or rotation rate of HST, including the scenario in which the HST batteries have died and HST is tumbling.

The next section gives an overview of the mathematical terms. Then the nonlinear estimation algorithm is summarized, followed by a summary of the nonlinear control algorithm. We then present the results of several scenarios, applied to the HST-HRV mission concept. Finally conclusions are given along with future considerations.

\section{Attitude and Angular Rate Definitions}

The attitude of a spacecraft can be represented by a quaternion, consisting of a rotation angle and unit rotation vector $e$, known as the Euler axis, and a rotation $\phi$ about this axis so that ${ }^{9}$

$$
q=\left[\begin{array}{c}
e \sin \left(\frac{\phi}{2}\right) \\
\cos \left(\frac{\phi}{2}\right)
\end{array}\right]=\left[\begin{array}{l}
\varepsilon \\
\eta
\end{array}\right]
$$

where $q$ is the quaternion, partitioned into a vector part, $\varepsilon$, and a scalar part, $\eta$. The target attitude quaternion is designated as $\boldsymbol{q}_{t}$, which defines the rotation from inertial to the target spacecraft body coordinates. The chase vehicle attitude quaternion is designated as $\boldsymbol{q}_{c}$.

The rotation, or attitude, matrix can be computed from the quaternion components as ${ }^{9}$

$$
R=R(q)=\left(\eta^{2}-\varepsilon^{T} \varepsilon\right) \mathrm{I}_{3}+2 \varepsilon \varepsilon^{T}-2 \eta S(\varepsilon)
$$

where $\mathrm{I}_{3}$ is a $3 \times 3$ identity matrix and $S(\varepsilon)$ is a matrix representation of the vector cross product operation.

$$
S(\varepsilon)=\left[\begin{array}{ccc}
0 & -\varepsilon_{z} & \varepsilon_{y} \\
\varepsilon_{z} & 0 & -\varepsilon_{x} \\
-\varepsilon_{y} & \varepsilon_{x} & 0
\end{array}\right]
$$

Note also that $R(\boldsymbol{q}) \varepsilon=\varepsilon$. The derivative of $R(\boldsymbol{q})$ is given as ${ }^{9}$

$$
\dot{R}(\boldsymbol{q})=-S(\boldsymbol{\omega}) R(\boldsymbol{q})
$$

where $\omega$ is the angular velocity in body coordinates.

A relative rotation between coordinate frames is computed as ${ }^{10}$

$$
\tilde{\boldsymbol{q}}_{r e l}=\left[\begin{array}{c}
\tilde{\varepsilon} \\
\tilde{\eta}
\end{array}\right]=\boldsymbol{q}_{1} \otimes \boldsymbol{q}_{2}^{-1}=\left[\begin{array}{cc}
\eta_{2} I-S\left(\varepsilon_{2}\right) & -\varepsilon_{2} \\
\varepsilon_{2}^{T} & \eta_{2}
\end{array}\right] \cdot\left[\begin{array}{l}
\varepsilon_{1} \\
\eta_{1}
\end{array}\right]
$$

Using the definition given in equation 2 , the relative attitude quaternion from the chase vehicle body coordinates to the target body coordinates is then

$$
\tilde{\boldsymbol{q}}_{c t}=\boldsymbol{q}_{t} \otimes \boldsymbol{q}_{c}^{-1}
$$

The angular velocity of the target vehicle body coordinates with respect to inertial space, resolved in target body coordinates, is designated as $\omega_{t}$. Similarly the angular velocity of the chase vehicle in chase vehicle body coordinates is designated as $\omega_{c}$.

\section{Target Vehicle Nonlinear Angular Velocity Estimator}

The angular velocity estimator is intended for the scenario in which the target vehicle is non-cooperative. For example, in the HST robotic servicing mission the estimator would be used in the event that the HST batteries have died and no telemetry is available from HST. The chase vehicle is equipped with an accurate quaternion star tracker, which provides $q_{c}$, and a sensor system which produces a measurement of the relative quaternion, $\vec{q}_{c t}$. The unknown target vehicle angular velocity is estimated in the inertial coordinate system through the estimation of the target vehicle inertial angular momentum. The target vehicle angular velocity 
in body coordinates is computed by a transformation of the inertial angular velocity. The development and stability analysis of the algorithm are provided in reference 7 . The algorithm is summarized here.

The system equations consist of the kinematic equation for the target vehicle attitude quaternion and Euler's equation for the target vehicle given in inertial coordinates

$$
\begin{gathered}
\dot{\boldsymbol{q}}_{t}=\frac{1}{2} Q\left(\boldsymbol{q}_{t}\right) \omega_{t}=\frac{1}{2} Q\left(q_{t}\right) R_{t} \omega_{i, t}=\frac{1}{2} Q\left(q_{t}\right) I_{\dot{t}}^{-1} R_{t} \boldsymbol{h}_{i, t} \\
\dot{h}_{i, t}=T_{i, t}
\end{gathered}
$$

where $I_{t}$ is the target vehicle inertia matrix in body coordinates, assumed to be constant. Note that $I_{i, t}=R_{t}^{T} I_{t} R_{t}$, where $I_{i, t}$ is the inertia matrix in inertial coordinates and $R_{t}$ is the target vehicle attitude matrix defining the transformation from inertial to target body coordinates. $T_{i, t}$ is the external torque acting on the target vehicle, resolved in inertial coordinates, and

$$
Q\left(\boldsymbol{q}_{t}\right)=\left[\begin{array}{c}
\eta_{t} \mathrm{I}_{3}+S\left(\varepsilon_{t}\right) \\
-\varepsilon_{t}^{T}
\end{array}\right]=\left[\begin{array}{c}
Q_{1}\left(\boldsymbol{q}_{t}\right) \\
-\varepsilon_{t}^{T}
\end{array}\right]
$$

where, by inspection, $Q_{1}\left(\boldsymbol{q}_{t}\right)=\eta_{t} \mathbf{I}_{3}+S\left(\varepsilon_{t}\right)$.

The predicted target vehicle quaternion as defined as

$$
\hat{\boldsymbol{q}}_{t}=\left[\begin{array}{c}
\hat{\varepsilon}_{t} \\
\hat{\eta}_{t}
\end{array}\right]
$$

The attitude error is defined as the relative orientation between the predicted attitude $\hat{q}_{t}$ and the measured attitude, $\boldsymbol{q}_{t}$, computed from equation 2 from the measured relative attitude quaternion and the measured chase vehicle attitude quaternion. The estimator attitude error is

$$
\tilde{q}_{t}=\left[\begin{array}{c}
\tilde{\varepsilon}_{t} \\
\tilde{\eta}_{t}
\end{array}\right]=q_{t} \otimes \hat{q}_{t}^{-1}
$$

The state estimators for the HST attitude and angular momentum are defined as

$$
\begin{gathered}
\dot{\hat{q}}_{t}=\frac{1}{2} Q\left(\hat{q}_{t}\right) R\left(\tilde{q}_{t}\right)^{T}\left[I_{t}^{-1} R_{t} \hat{\boldsymbol{h}}_{i, t}+k \tilde{\varepsilon}_{t} \operatorname{sign}\left(\tilde{\eta}_{t}\right)\right] \\
\dot{\hat{h}}_{i, t}=T_{i, t}+\frac{\beta}{2} R_{t}^{T} I_{t}^{-1} \tilde{\varepsilon}_{t} \operatorname{sign}\left(\tilde{\eta}_{t}\right)
\end{gathered}
$$

The term $R\left(\tilde{\boldsymbol{q}}_{t}\right)^{T}$.in equation 6 transforms the angular velocity terms from the body frame to the predicted attitude frame. The gain $\mathrm{k}$ is chosen as a positive constant. Similarly, the learning rate, $\beta$, is also a positive constant. Essentially, $\hat{q}_{t}$ is a prediction of the attitude at time $t$, propagated with the kinematic equation using the estimated angular momentum.

The error equations are given as

$$
\dot{\tilde{\boldsymbol{q}}}_{t}=\frac{1}{2} Q\left(\tilde{\boldsymbol{q}}_{t}\right)\left(I_{t}^{-1} R_{t} h_{i, t}-I_{t}^{-1} R_{t} \hat{h}_{i, t}-k \tilde{\varepsilon}_{t} \operatorname{sign}\left(\tilde{\eta}_{t}\right)\right)
$$

Let $\tilde{h}_{i, t}=h_{i, t}-\hat{h}_{i, t}$. The derivative of $\tilde{h}_{i, t}$ is

$$
\dot{\tilde{h}}_{i, t}=-\frac{\beta}{2} R_{t}^{T} I_{t}^{-1} \tilde{\varepsilon}_{t} \operatorname{sign}\left(\tilde{\eta}_{t}\right)
$$

Note'that the equilibrium states for 8 and 9 are

$$
\left[\begin{array}{cc}
\tilde{\boldsymbol{q}}_{t}^{T} & \tilde{\boldsymbol{h}}_{i, t}^{T}
\end{array}\right]=\left[\begin{array}{lllllll}
0 & 0 & 0 & \pm 1 & 0 & 0 & 0
\end{array}\right]
$$

In the absence of any errors, equations 8 and 9 are exponentially stable, i.e $\hat{\omega}_{t} \rightarrow \omega_{t}$ exponentially fast. ${ }^{7}$ 
Reference 7 examines the stability of the nonlinear estimator given errors in the relative attitude quaternion. The measurement of the relative attitude quaternion from the vision and feature based sensor was the largest source of error for HRV. When the true quaternion is unknown, equations 6 and 7 cannot be implemented. Instead, the erroneous measured attitude $q_{t, m}$ is used in place of $q_{t}$, resulting in

$$
\begin{gathered}
\dot{\hat{q}}_{t}=\frac{1}{2} Q\left(\hat{q}_{t}\right) R\left(\tilde{q}_{t_{i} m}\right)^{T}\left[I_{v}^{-1} R_{t, m} \hat{h}_{i, t}+k \tilde{\varepsilon}_{t, m} \operatorname{sign}\left(\tilde{\eta}_{t, m}\right)\right] \\
\dot{\hat{h}}_{i, t}=\hat{T}_{i_{i} t}+\frac{\beta}{2} R_{t, m}^{T} I_{t}^{-1} \tilde{\varepsilon}_{t, m} \operatorname{sign}\left(\tilde{\eta}_{t, m}\right)
\end{gathered}
$$

$\hat{T}_{i, t}$ is the estimated external torque. The estimator, however, remains robust to disturbances in the relative attitude measurement. The addition of a leakage term ensures that the system remains bounded in the event of unusually large disturbances.

\section{Chase Vehicle Control Algorithm}

Prior to docking with the target vehicle, the chase vehicle control system must force the chase vehicle to match the attitude and attitude rates of the target vehicle to within some mission specific tolerance. In the non-cooperative scenario considered here, the target vehicle attitude and rates are provided by the nonlinear estimator of the previous section. The chase vehicle is equipped with star trackers and calibrated gyros to provide the necessary feedback signals to the control algorithm. In this work we consider the nonlinear adaptive controller of reference 8 .

The attitude dynamics of the chase vehicle, modelled as a rigid spacecraft, are given as (the time dependence is omitted for clarity)

$$
I_{c} \dot{\omega}_{c}-S\left(I_{c} \boldsymbol{\omega}_{c}+\boldsymbol{h}_{c}\right) \boldsymbol{\omega}_{c}=\boldsymbol{u}+\dot{\boldsymbol{h}}_{c}
$$

where $I_{c}$ is the inertia matrix, $\boldsymbol{u}$ is the applied external torque, $\omega_{c}$ is the angular velocity, and $h_{c}$ is the wheel momentum in body coordinates. The goal of the control law is to force the attitude of the chase vehicle, $\boldsymbol{q}_{c}$ to asymptotically track the target vehicle attitude, $q_{t}$, and the target vehicle rate, $\omega_{t}$. The attitude tracking error is computed with equation 2 as

$$
\tilde{q}_{t c}=\left[\begin{array}{c}
\tilde{\varepsilon}_{t c} \\
\tilde{\eta}_{t c}
\end{array}\right]=q_{c} \otimes q_{t}^{-1}
$$

The rate tracking error is given as

$$
\tilde{\omega}_{t c}=\omega_{c}-R\left(\tilde{\boldsymbol{q}}_{t c}\right) \boldsymbol{\omega}_{t}
$$

where $R\left(\tilde{\boldsymbol{q}}_{t c}\right)$ transforms the angular velocity from the target vehicle body frame to the chase vehicle body frame.

The control law is given as

$$
\boldsymbol{u}+\dot{\boldsymbol{h}}_{\mathrm{c}}=-K_{D} s(t)+I_{c} \alpha_{r}-S\left(I_{c} \omega_{c}+\boldsymbol{h}_{c}\right) \boldsymbol{\omega}_{r}
$$

$K_{D}$ is any symmetric, positive definite matrix and $s$ is an error defined as

$$
s=\tilde{\omega}_{t c}+\lambda \tilde{\varepsilon}_{t c}=\omega_{c}-\omega_{r}
$$

where $\lambda$ is any positive constant. The reference angular velocity $\omega_{r}$ is computed as

$$
\omega_{r}=R\left(\tilde{q}_{t c}\right) \omega_{t}-\lambda \tilde{\varepsilon}_{t c}
$$

The derivative of $\omega_{r}$ is given as

$$
\alpha_{r}=\dot{\omega}_{r}=R\left(\tilde{\boldsymbol{q}}_{t c}\right) \dot{\boldsymbol{\omega}}_{t}-S\left(\tilde{\boldsymbol{\omega}}_{t c}\right) R\left(\tilde{\boldsymbol{q}}_{t c}\right) \omega_{t}-\lambda Q_{1}\left(\tilde{\boldsymbol{q}}_{c t}\right) \tilde{\boldsymbol{\omega}}_{t c}
$$

Asymptotically perfect tracking is obtained with the above control scheme, given noise free measurements of the states $\omega_{c}$ and $q_{c}$. Reference 11 also shows that the control scheme is robust to gyro bias errors and 
bounded noise disturbances. (The gyros are assumed to be calibrated for scale factor and misalignment errors prior to the approach and capture phase. Gyro bias errors can be estimated a priori as well.)

In a typical control application the desired states are well defined. In this work, however, the desired states are estimated with the nonlinear estimator outlined in the previous section. The nonlinear estimator provides continuous estimates of the desired attitude, desired rate, and derivative of the desired rate. The desired attitude is provided by the estimator as $\hat{\boldsymbol{q}}_{t}$. The control error is then computed as

$$
\tilde{\boldsymbol{q}}_{t c}=\left[\begin{array}{c}
\tilde{\boldsymbol{\varepsilon}}_{t c} \\
\tilde{\eta}_{t c}
\end{array}\right]=\boldsymbol{q}_{c} \otimes \hat{\boldsymbol{q}}_{t}^{-1}
$$

The desired rate, $\omega_{t}$ in the target body coordinates, is computed from the estimated angular momentum as

$$
\omega_{t}=I_{t}^{-1} R\left(\hat{q}_{t}\right) \hat{h}_{i, t}
$$

The chase vehicle desired angular acceleration, in the target body coordinates, is then

$$
\dot{\omega}_{t}=I_{t}^{-1}\left[\dot{R}\left(\hat{\boldsymbol{q}}_{t}\right) \hat{\boldsymbol{h}}_{i, t}+R\left(\hat{\boldsymbol{q}}_{t}\right) \dot{\hat{\boldsymbol{h}}}_{i, t}\right]
$$

Substituting for $\dot{R}\left(\hat{\boldsymbol{q}}_{t}\right)$ from equation 1 and $\dot{\hat{h}}_{i, t}$ from equation $11, \dot{\omega}_{t}$ is written as

$$
\dot{\boldsymbol{\omega}}_{t}=I_{t}^{-1}\left[S\left(\hat{\boldsymbol{\omega}}_{t}\right) R\left(\hat{\boldsymbol{q}}_{t}\right) \hat{\boldsymbol{h}}_{i, t}+R\left(\hat{\boldsymbol{q}}_{t}\right)\left(\hat{T}_{i, t}+\frac{\beta}{2} R_{t, m}^{T} I_{t}^{-1} \tilde{\varepsilon}_{t, m} \operatorname{sign}\left(\tilde{\eta}_{t, m}\right)\right)\right]
$$

where $\tilde{\varepsilon}_{t, m}, \tilde{\eta}_{t, m}$, and $R_{t, m}$ are all calculated using the measured attitude. The goal is to demonstrate that the control algorithm will asymptotically track the estimated. states, which are bounded estimates of the true states in the presence of measurement errors.

\section{Simulation Results}

The algorithms outlined in the previous sections are tested in two simulation environments. Both simulations are based on an HRV-HST rendezvous scenario. The first simulation is developed in Matlab. The Matlab simulation gives a high level indication of the performance of the combined estimator and control algorithm. Then the simulation is developed in the NASA Goddard Space Flight Center simulation environment known as Freespace (FSP). FSP is a C-based high performance, simulation environment. FSP utilizes shared memory with a modular environment which allows for simultaneous processing and visualization. The results from the Matlab simulation are presented first, followed by results from FSP.

The HST (target) inertia is ${ }^{12}$

$$
I_{t}=\left[\begin{array}{ccc}
36046 & -706 & 1491 \\
-706 & 86868 & 449 \\
1491 & 449 & 93848
\end{array}\right] \mathrm{kg} \cdot \mathrm{m}^{2}
$$

The HRV (chase) inertia matrix is ${ }^{12}$

$$
I_{c}=\left[\begin{array}{ccc}
18748 & 525 & -2197 \\
525 & 55903 & 1366 \\
-2197 & 1366 & 53025
\end{array}\right] \mathrm{kg} \cdot \mathrm{m}^{2}
$$

The HST truth model simulation includes aerodynamic torque and gravity gradient torque with second order effects. ${ }^{13,14}$

The HST attitude measurements are simulated by rotating the true HST attitude by a random angle about a random unit direction. The random angle is generated from a zero mean, normal distribution, the random unit direction is generated from a zero mean, uniform distribution. The standard deviation of the random angle is first set at 5 degrees, after approximately 30000 seconds the standard deviation is reduced to 1 degree. The vision sensors are expected to improve as the separation between the vehicles decreases. The estimator is initialized with the first attitude measurement. The HRV attitude is initialized at $\boldsymbol{q}_{c}=[0,1,0,0]$. 
The initial HRV angular velocity is $\omega_{c}=[0,0,0] \mathrm{deg} / \mathrm{sec}$. The HST truth model is initialized with a random attitude, and a random initial angular velocity, generated with a zero mean, normal distribution with a standard deviation of $0.07 \mathrm{deg} / \mathrm{sec} /$ axis.

Figures 1 through 4 shows the true attitude control error and the angular velocity control error for each axis for 100 test cases. Each case contains a different initial random attitude and rate in the HST truth model, and different random measured attitudes. The top plot of each figure shows the convergence once the controller is started at 5000 seconds. The bottom plot shows the steady state behavior. The final
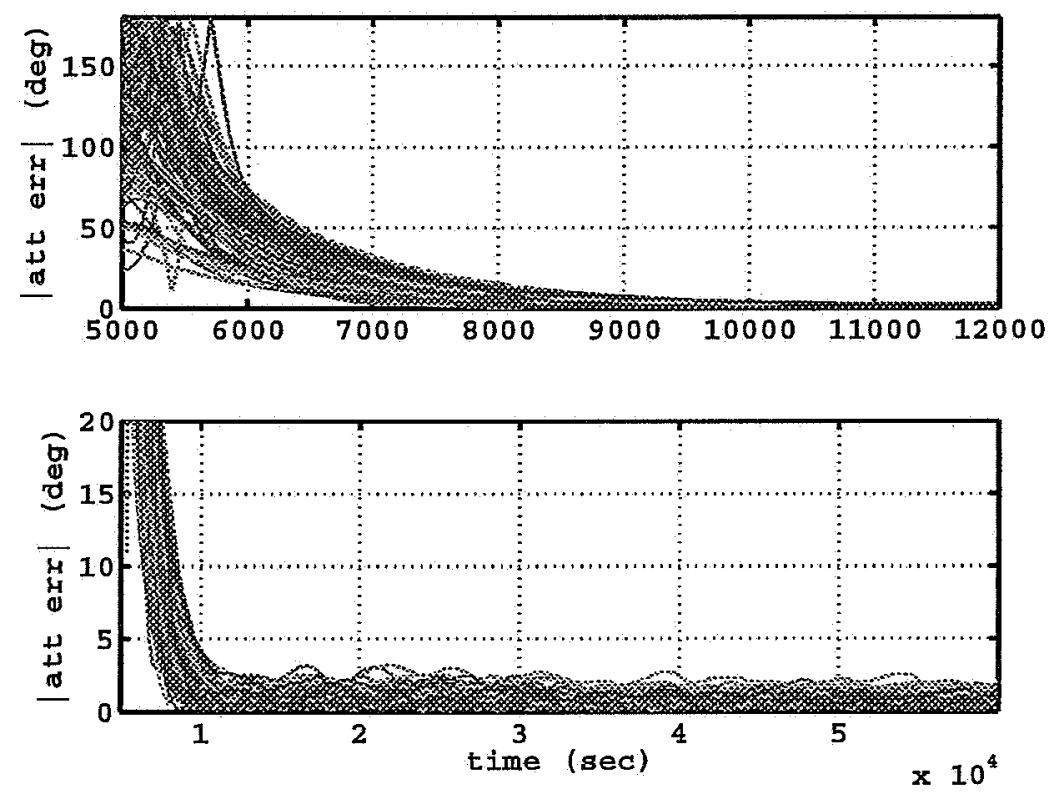

Figure 1. True Attitude Control, Transient and Steady State Errors
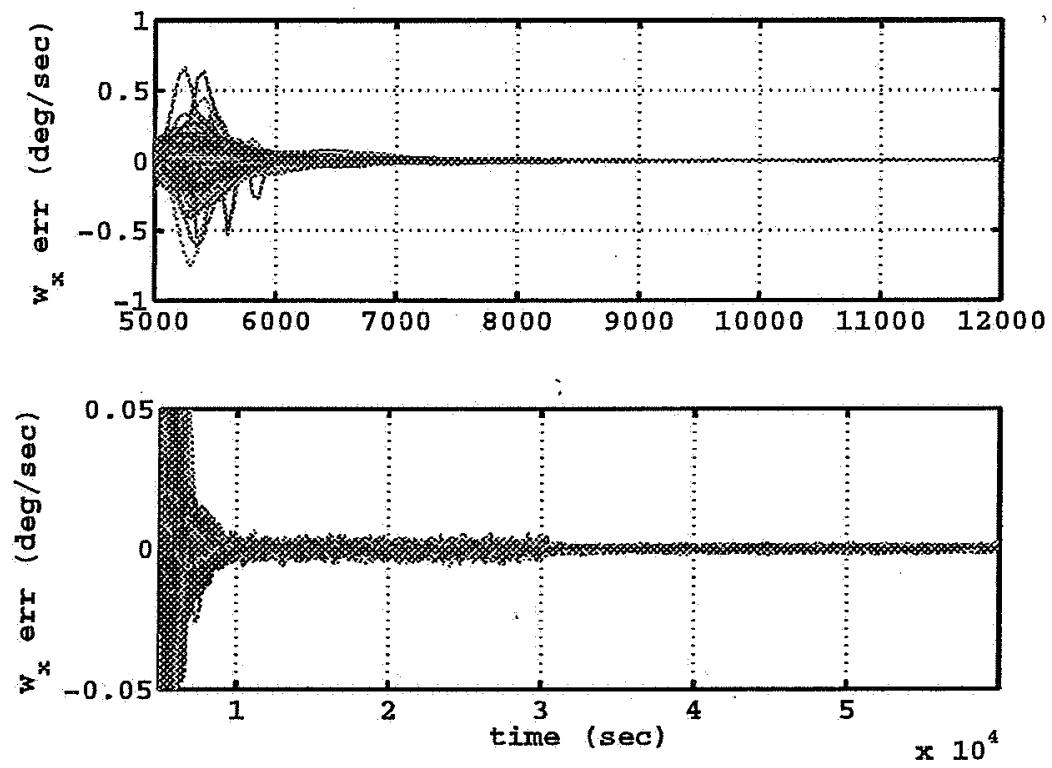

Figure 2. True X Axis Angular Velocity Control Errors, Transient and Steady State

average attitude control error for all 100 cases is $0.37 \mathrm{deg}$. The final average angular velocity control error 

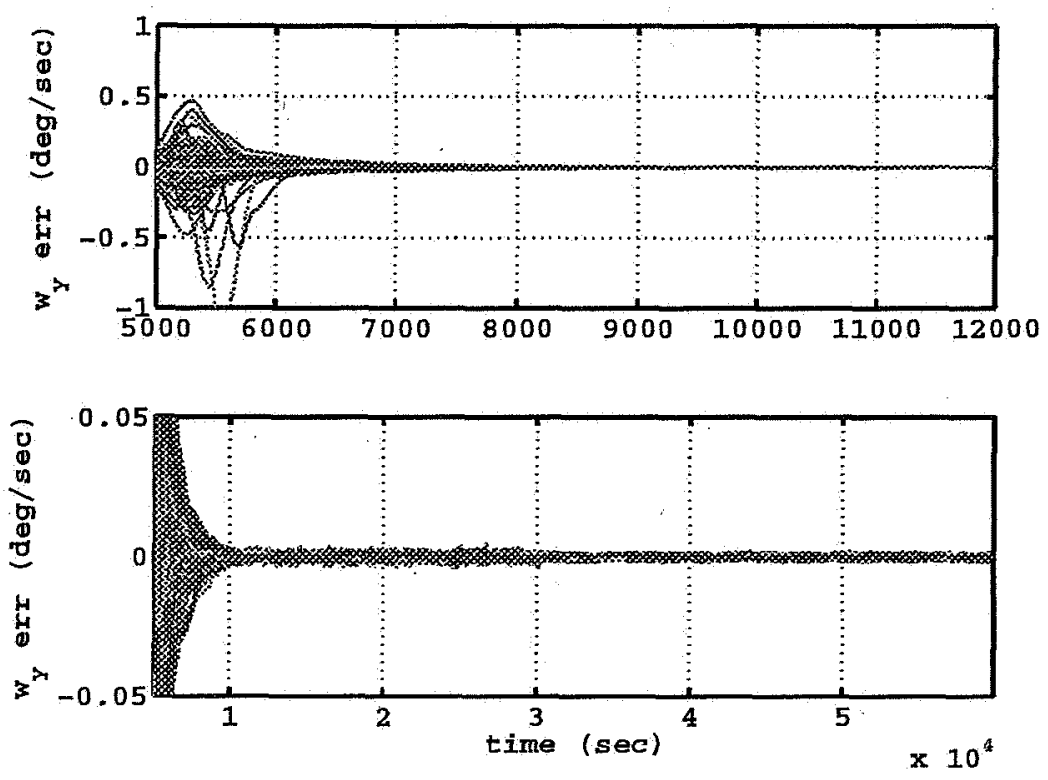

Figure 3. True $Y$ Axis Angular Velocity Control Errors, Transient and Steady State
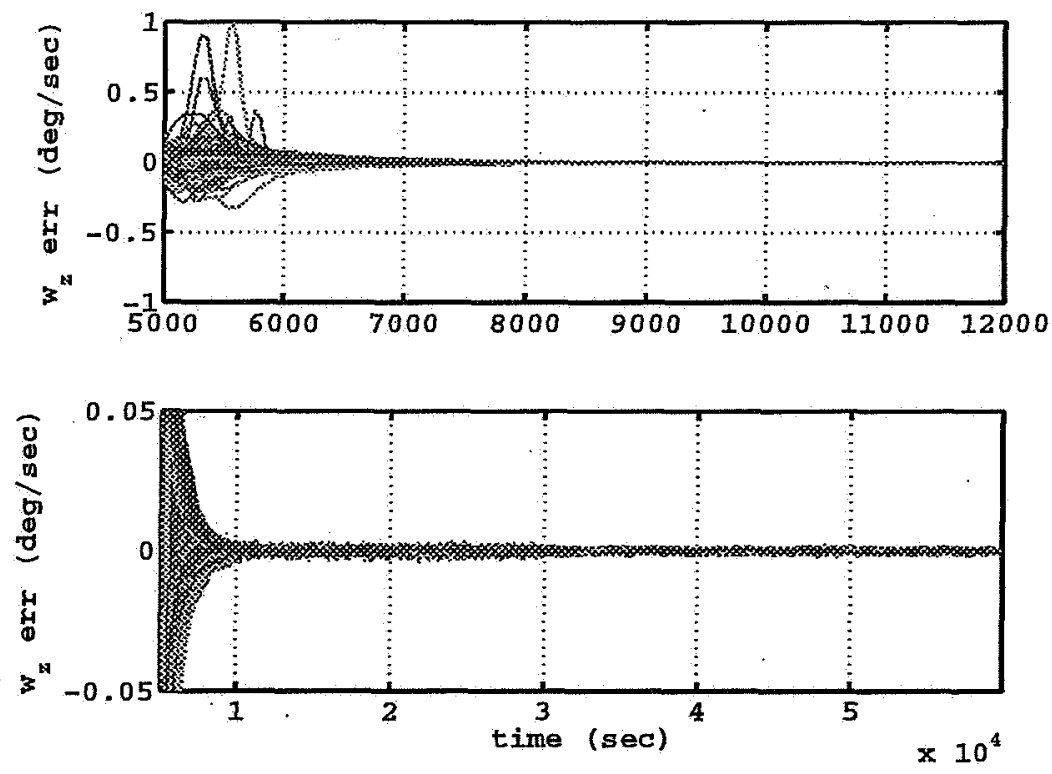

Figure 4. True Z Axis Angular Velocity Control Errors,-Transient and Steady State 
for all 100 cases is

$$
\tilde{\omega}_{t c}(\text { ave in } \operatorname{deg} / \mathrm{sec})=\left[\begin{array}{c}
-0.00028 \\
0.0062 \\
-0.0072
\end{array}\right]
$$

Next, the algorithms are tested in the FSP high fidelity simulator. The Freespace truth model contains gravity gradient and aerodynamic external torques acting on both HST and HRV. The HRV gravity gradient torques are input to the control algorithm as a feed forward torque. The scenario developed for these results places the HRV at the $50 \mathrm{~m}$ hold point on the HST -V1 axis (boresight axis), which is the point at which the rescue mission would have begun to match the attitude and angular rate of HST for docking.

The estimator performance in Freespace compares to the results in reference 7, though with a longer convergence time. The attitude of HRV is initially controlled using a coarse attitude control with no roll constraint, until $15000 \mathrm{sec}(4 \mathrm{~h}, 10 \mathrm{~min})$ when the target attitude tracking controller is enabled. Figures 5 and 6 show the magnitude of the true attitude control error and the true angular rate error. The magnitudes of the attitude control error converge to $0.02 \mathrm{deg}$ at the end but vary as high as $0.15 \mathrm{deg}$. The rate error does not have as much variation and converges to $2.8 \times 10^{-5} \mathrm{deg} / \mathrm{sec}$. Next, sensor noise is added to the relative

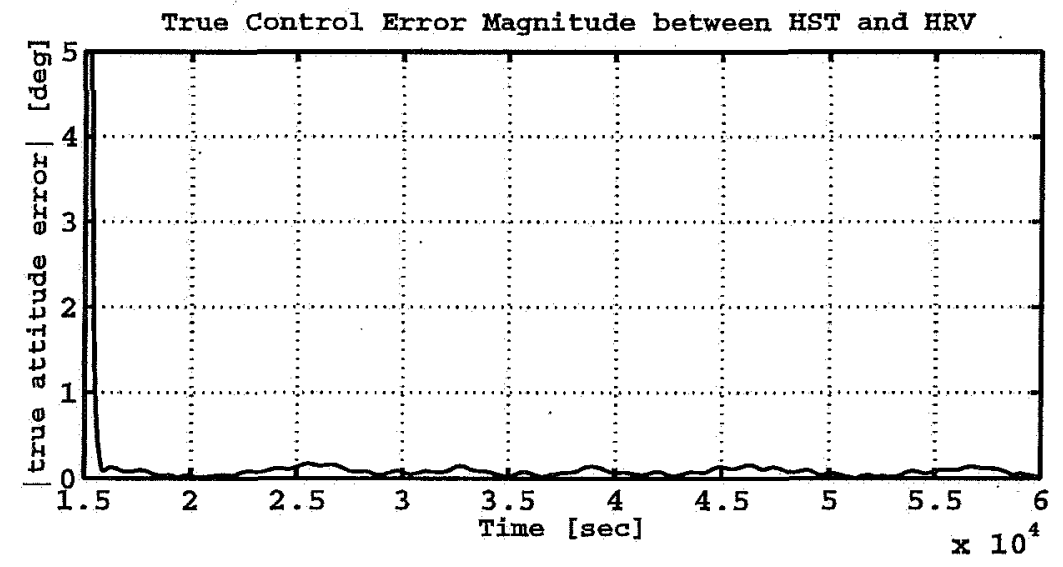

Figure 5. True Attitude Control Errors from Freespace, No Measurement Errors

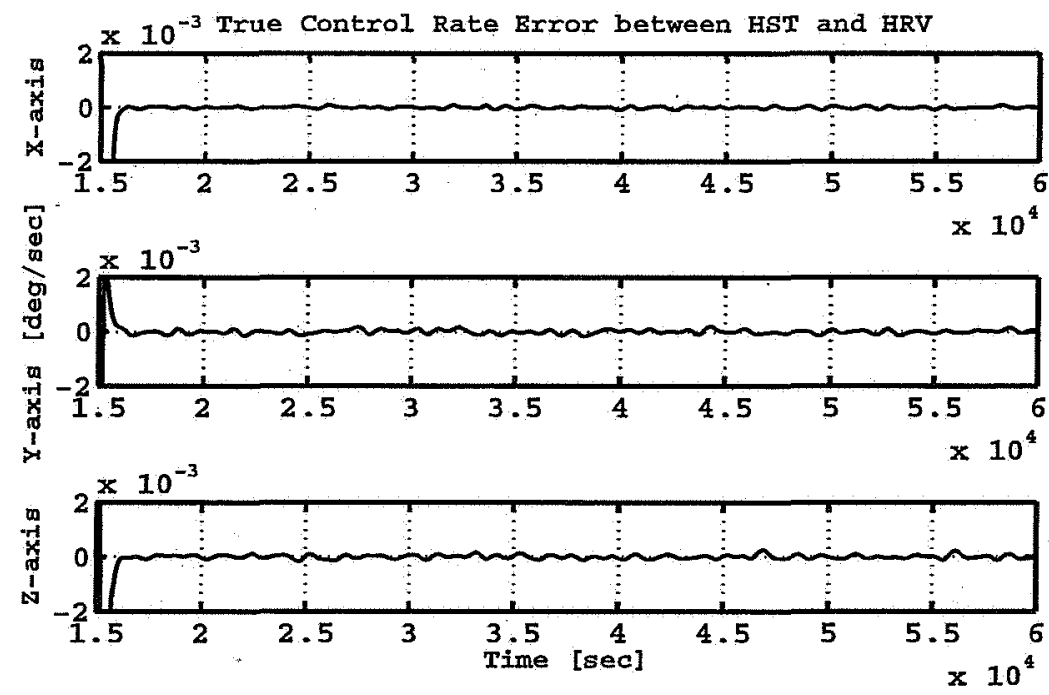

Figure 6. True Rate Control Errors from Freespace, No Measurement Errors 
quaternion measurement by corrupting the true relative attitude measurement with a randomly generated quaternion with a $3 \sigma$ magnitude of $10 \mathrm{deg}$. Figure 7 shows the magnitude of the true attitude controller error, which converges to $0.75 \mathrm{deg}$, with only slight variations above $1 \mathrm{deg}$. Figure 8 shows the convergence of the true angular rate error, which converges to $0.00091 \mathrm{deg} / \mathrm{sec}$. For a relative quaternion measurement with only $1 \mathrm{deg}$ of error, the controller attitude and rate errors converge to $0.075 \mathrm{deg}$ and $0.0024 \mathrm{deg} / \mathrm{sec}$, respectively.

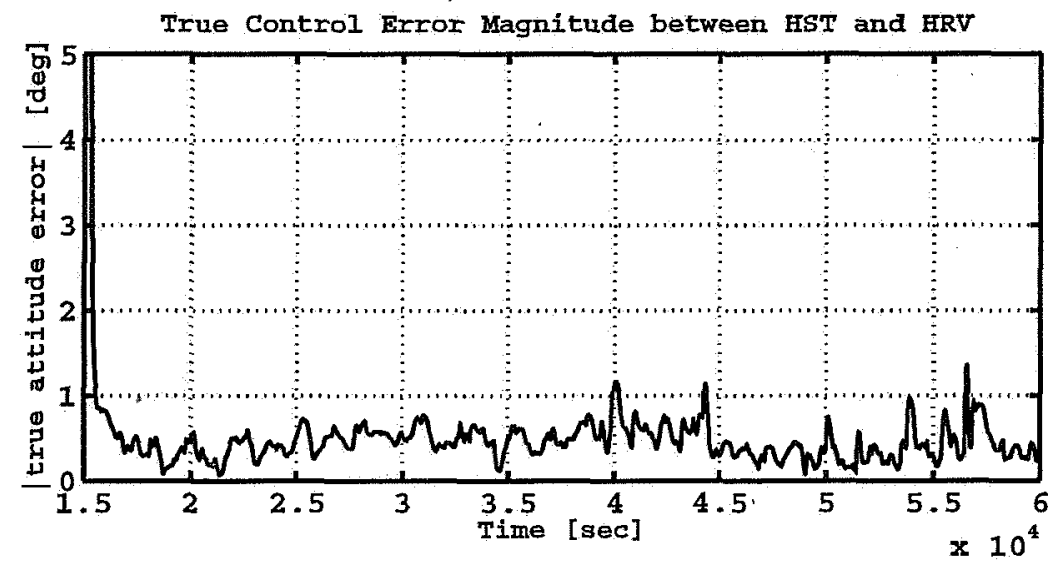

Figure 7. True Attitude Control Errors from Freespace, 10 deg Measurement Error

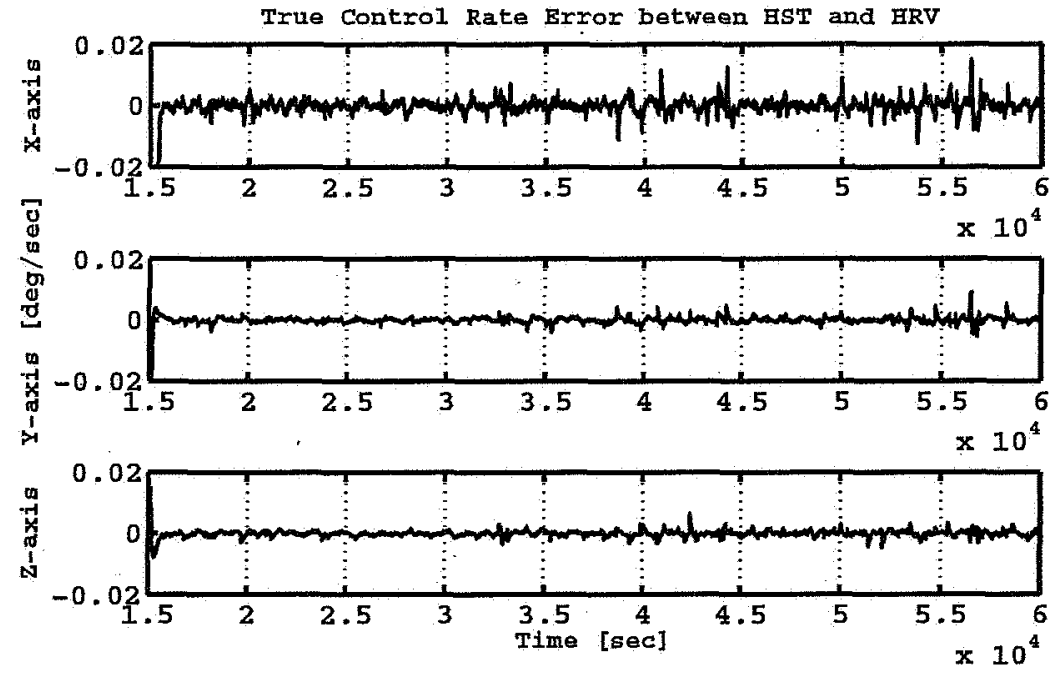

Figure 8. True Rate Control Errors from Freespace, $10 \mathrm{deg}$ Measurement Error

These results confirm the findings of the low fidelity MATLAB simulation, and also enable further experiments to characterize the estimator / controller interaction and behavior under various conditions (i.e. varying the measurement error, the initial target rates, and dynamic model fidelity).

\section{Conclusions}

An approach for estimating the attitude and rates of a non-cooperative target vehicle and then controlling the chase vehicle to match the estimated attitude and rates is presented. The attitude and rates are estimated with a nonlinear estimation algorithm that is robust to measured attitude errors. The nonlinear control algorithm is asymptotically stable in the absence of errors, and remains robust given gyro measurement 
errors. The algorithms are applied to the proposed HST robotic servicing mission and are tested first with a Matlab simulation which includes random attitude measurements and random initial conditions. A Monte Carlo simulation demonstrates that the combined algorithms are stable and converge to less than 0.4 deg and $0.01 \mathrm{deg} / \mathrm{sec}$ in attitude and rate control errors (magnitude), respectively. The algorithms are then tested in a high performance simulation environment known as Freespace. Again, the combined algorithms are stable and converge to final errors less than $0.075 \mathrm{deg}$ and $0.0024 \mathrm{deg} / \mathrm{sec}$.

Future work will expand the simulation scenario in Freespace. The vision sensors will be modelled and used to provide the relative attitude measurement. Additional fidelity will be added to the wheel models and the chase vehicle gyros, along with a star tracker based attitude estimation algorithm for the chase vehicle.

\section{References}

\footnotetext{
${ }^{1}$ Machula, M. F. and Sandhoo, G. S., "Rendervous and Docking for Space Exploration," 1st Space Exploration Conference: Continuing the Voyage of Discovery, No. 2005-2716, Orlando, Florida, January 2005.

${ }^{2}$ Zimpfer, D., Kachmar, P., and Tuohy, S., "Autonomous Rendezvous, Capture and In-Space Assembly: Past, Present, and Future," 1st Space Exploration Conference: Continuing the Voyage of Discovery, No. 2005-2523, Orlando, Florida, January 2005.

"Wingo, D. R., "Orbital Recovery's Responsive Commercial Space Tug for Life Extension Missions," AIAA 2nd Responsive Space Conference, No. 2004-6118, San Diego, California, January 2004.

${ }^{4}$ Bounova, G. A., Mierry, T. D., and deWeck, O. L., "Search Algorithms for Space Tug Rendezvous: Simulation and Experiment," 2004 IEEE Aerospace Conference, Big Sky, Montana, March 2004.

${ }^{5}$ Kimura, S., Nagai, Y., Yamamoto, H., Masuda, K., and Abe, N., "Approach for On-Orbit Maintenance and Experiment Plan Using 150kg-class Satellites," 2005 IEEE Aerospace Conference, Big Sky, Montana, March 2005.

"Hubble Space Telescope Program, "HST Robotic Servicing Mission Concept Review," Held at NASA Goddard Space Flight Center, May 2004.

${ }^{7}$ Thienel, J. K., Queen, S. Z., VanEepoel, J. M., and Sanner, R. M., "Hubble Space Telescope Angular Velocity Estimation During the Robotic Servicing Mission," AIAA Guidance, Navigation, and Control Conference, No. 2005-6396, San Francisco, California, August 2005.

${ }^{8}$ Egeland, O. and Godhavn, J., "Passivity-Based Adaptive Attitude Control of a Rigid Spacecraft," IEEE Transactions on Automatic Control, Vol. 39, No. 4, April 1994, pp. 842-846.

${ }^{9}$ Shuster, M. D., "A Survey of Attitude Representations," The Journal of Astronautical Sciences, Vol. 41, No. 4, OctoberDecember 1993, pp. 439-517.

${ }^{10}$ Sanner, R. M., "Adaptive Attitude Control Using Fixed and Dynamically Structured Neural Networks," AIAA Guidance, Navigation, and Control Conference, No. 96-3891, San Diego, California, July 1996.

${ }^{11}$ Thienel, J., Nonlinear Observer/Controller Designs for Spacecraft Attitude Control Systems with Uncalibrated Gyros, Ph.D. thesis, University of Maryland, 2004. 2004.

${ }^{12}$ Queen, S., "HRV GNC Peer Review, Flight Performance Analysis," Tech. rep., NASA Goddard Space Flight Center,

${ }^{13}$ Skelton, E. and Ramsey, P., "HST Aerodynamic Torque Model and Algorithm Description Document," Tech. rep., Mission Operations, Systems Engineering and Software Program, Lockheed Martin Corporation - Space Systems Company, 2004.

${ }^{14}$ Roithmayr, C. M., "Gravitational Moment Exerted on a Small Body by an Oblate Body," AIAA Journal of Guidance, Control, and Dynamics, Vol. 12, No. 3, May-June 1989, pp. 441-444.
} 